\title{
Investigating an all-hydraulic hybrid system for diesel-hydraulic rail cars
}

\author{
Martin Kache \\ Received: 14 February 2013 / Accepted: 20 September 2013 / Published online: 5 October 2013 \\ (C) The Author(s) 2013. This article is published with open access at SpringerLink.com
}

\begin{abstract}
Purpose This paper outlines a complex simulation model for parallel-hybrid diesel railcars with hydrodynamic power transmission. It contributes to the discussion concerning whether a hydrostatic recuperation system can be an alternative to electric systems using double-layer capacitors or flywheels. The paper focusses on a hybrid system with realistic parameters concerning mass, power, and energy content that should be applicable to both existing and newly built vehicles.

Methods A simulation process that is based on the 1-d-multidomain simulation tool Imagine.Lab AMESim is presented. The simulation comprises a conventional and an alternative drive train as well as the longitudinal dynamics of the vehicle along with the control of the vehicle motion. Energy-efficient driving techniques and timetable restrictions are taken into account when comparing train runs of different drivetrain configurations.

Results Simulations based on real route data for eight different railway lines show a reduction of fuel consumption between 5 and $16 \%$ due to the hydrostatic recuperation of energy. Station spacing and mean line gradients prove to be important line-side factors impacting fuel economy.

Conclusions Hydrostatic recuperation represents a feasible solution for railcars, provided that possible spatial and economic obstacles can be overcome. As the simulation results are promising, further hybrid configurations will be considered for simulation. A comprehensive comparison to models of hybrid diesel railcars using different energy storages is the next step.
\end{abstract}

Keywords Railcar · Parallel-hybrid · Fuel consumption · Diesel-hydraulic power transmission · Pressure storage · Hydraulic accumulator · Hydrostatic recuperation · System simulation

\footnotetext{
M. Kache $(\bowtie)$

Rail Vehicle Technology, TU Dresden, Hettnerstraße 1-3,

01069 Dresden, Germany

e-mail: Martin.Kache@tu-dresden.de
}

\section{Introduction}

The introduction of hybrid drivetrains is a promising means of reducing the fuel consumption of railcars. The question whether an electric or a non-electric system should be preferred for the recuperation of energy is not straightforward as there are multiple aspects to be taken into account. The additional demand of mass and installation space as well as the capability of the hybrid system in terms of power and energy content play an important role. Further important aspects worth considering include the cost, availability, longevity, and safety of the chosen technology. All storage technologies that are available today necessitate compromises as none of them are able to comply with all given demands optimally.

A multitude of research on the hybridisation of railcars has been carried out [1-8] and continues. Fundamental investigations into the suitability of diesel railcars for hybridisation have been published by Hillmansen and Roberts [1], Nick [2], and $\mathrm{Lu}$ et al. [3]. All the publications cited have a high degree of abstraction in common and focus on the general energy flows within hybrid drive trains without modelling specific hybrid configurations. In addition to the investigations mentioned above, there have been some more detailed studies on hybrid diesel railcars with electric power transmission $[4,5]$ using electric energy storage devices. Detailed investigations into diesel railcars with hydraulic or hydromechanical power transmission have been provided by Read et al. [6], and Dittus et al. [7], respectively. The former included energy efficient driving strategies in their analysis, but remained rather vague with respect to a realistic dimension of the hybrid drivetrain. Dittus and Ungethüm, on the other hand, chose a realistic approach to estimate the power and energy content provided by the hybrid equipment, but paid less attention to the simulation of an energy-efficient driving style. Non-electric energy storage devices have not been considered by any of the authors cited, except for Read et. al. [6], who stated that flywheel storages could be a suitable alternative to electric double layer capacitors. 
This paper covers a sub-topic of a comprehensive research project on the hybridisation of diesel railcars with hydrodynamic power transmission operating on non-electrified branch and main lines. It aims at comparing parallel hybrid structures based on hydraulic accumulators to those based on electric energy storages. A realistic sizing of the hybrid drive train based on mass balances is taken into account. The simulations within this project are based on the route data of eight different real non-electrified railway lines from all over Germany. Energy saving driving strategies are also considered so as not to overestimate the effect of hybridisation on the fuel consumption. The research behind this paper has been triggered by the following questions: Can a mere hydraulic system be a feasible alternative when it comes to the hybridisation of railcars with hydrodynamic power transmission? What can be expected of such a system with regard to the energy efficiency of both the hybrid drivetrain and the fuel consumption of the vehicle?

A considerable part of the current fleet of railcars in Germany and Central Europe features hydrodynamic power transmission and has reached an age when a refurbishment not only of the interior equipment (e.g. seats and comfort features) but also of the drivetrains is worth considering. Keeping the drivetrains purely mechanical might offer advantages in maintenance and repair as well as reducing the cost for the conversion of the vehicle. Hydraulic hybrid systems have been developed for commercial road vehicles and some vehicles have successfully been introduced into daily service [9-11].

The basic layout of a hydrostatic hybrid drive train is shown in the next section, which is followed by a description of a simulation model that has been developed by the author. Some simulation results showing the effect of hybridisation on real non-electrified railway lines are discussed thereafter and the paper concludes by outlining the prospects of the research project that constitutes the framework of the simulations discussed here.

\section{Basic layout of the hybrid concept}

Three out of four classes of Deutsche Bahn's regional railcars with hydrodynamic power transmission consist of vehicles with two sections (see Fig. 1). German class 612 was chosen as a typical example of this kind of vehicle and served as an orientation when setting up the simulation model. The basic layout of these vehicles as well as some technical specifications can be found in Fig. 1. Configured to run on lines in a hilly environment with speeds of up to $160 \mathrm{~km} / \mathrm{h}$, the combined installed power of its diesel engines exceeds $1,000 \mathrm{~kW}$.

Due to the layout of a diesel-hydraulic drive line, the hybridisation of these railcars leads to a parallel hybrid configuration (see Fig. 2). In contrast to rail vehicles with electric power transmission, where the existing drivetrain "simply" has to be extended by an energy storage device, the situation here is more complex. The alternative traction equipment has to be linked to the conventional drivetrain via torque or speed-coupling elements resulting in solutions of different complexity [12]. Using a torque coupling in the form of a simple one-stage gear box in connection with shifting clutches is likely to be a reasonably priced solution and was therefore chosen for modelling.

As illustrated by Fig. 1, there are trailing bogies in the conventional vehicles that could be converted into driven ones. This leads to a second possible parallel hybrid architecture that is worth considering (see Fig. 2). Choosing between these two options will be strongly influenced by the acceptable complexity of the conversion as well as cost, space, and adhesion issues. Separating the wheelsets driven by the two different drive lines can provide considerable advantages concerning the transmission of tractive forces from wheel to rail. This is of some importance as it has to be kept in mind that the combined torque of the conventional and the alternative drive train have to be transferred via the same pairs of wheels in the case that the wheel arrangement is left untouched. This can result in increased sliding under suboptimal circumstances, thus influencing the performance of the hybrid vehicle negatively.

\section{Simulation model}

In order to investigate the effect of the hybridisation on the fuel consumption, a modular simulation model was developed at the Chair of Rail Vehicle Technology at TU Dresden [13]. This was accompanied by studying the behaviour of both the driver and the diesel-hydraulic powertrain in real-life operation [14].

The simulation model is based on Imagine.Lab AMESim, which is a powerful tool for one-dimensional multi-domain system simulation. There have been three phases of modelling, as illustrated in Fig. 3.

\subsection{Basic simulation model}

The core of the simulation is a basic model, representing the drive train of the conventional vehicle. The major elements developed comprise the diesel engine itself, the turbo transmission, the auxiliaries, as well as the vehicle and the resistance forces acting upon it. The models of the diesel engine and power transmission are based on measured characteristic diagrams and, concerning the hydraulic converter and couplings, semiempirical equations that have been provided by Voith Turbo. The fuel consumption is calculated for each time step depending on the diesel engine's torque and speed with the help of a fuel consumption map and summed up by an integration element. In addition to determining the fuel consumption, the model of the diesel engine is also prepared for the integration of maps representing the exhaust gas mass flow and the exhaust gas temperature respectively. These data are needed to 
Fig. 1 Outline of the railcar type the simulation model is based on

\section{Exemplary regional twin-section railcar (class 612)}

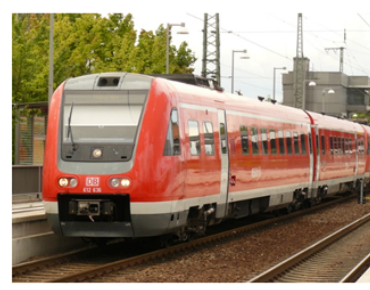

Technical specifications:

vehicle mass (2/3 of all seats taken): $\quad 106 \mathrm{t}$

maximum speed:

power of diesel engines:

type of fluid transmission:

wheel arrangement:

$60 \mathrm{~km} / \mathrm{h}$

$2 \times 559 \mathrm{~kW}$

converter+coupling+

coupling

$2^{\prime} B^{\prime}+B^{\prime} 2$

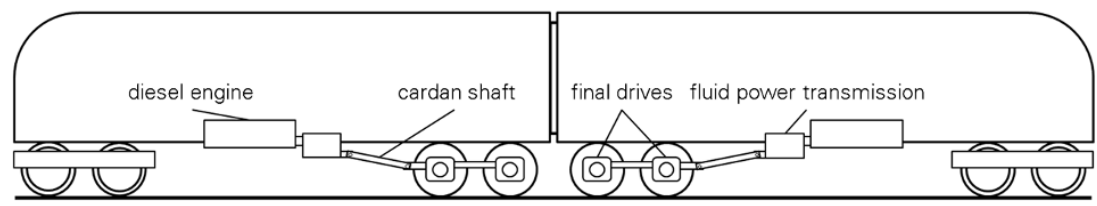

incorporate further submodels representing equipment to recover exhaust gas energy.

In case the traction power is switched off, the controller of the diesel engine adjusts the engine speed by using a separate torque-speed characteristic for idling. It can thus compensate the changing power demand of the auxiliaries by adjusting the torque. As soon as the controller detects the application of the brakes, the speed of the diesel engine is enhanced to a fixed value $(1,300 \mathrm{rev} / \mathrm{min})$. This is a characteristic behaviour of vehicles featuring a hydrodynamic brake and provides for a sufficient power supply to the cooling system in order to transfer the heat generated during the braking process.
The power requirement of the auxiliary equipment is also accounted for. A special submodel generates a certain pattern of power demand based upon empirical assumptions of the duty cycles of the four main contributors: the air compressor, the air conditioning and ventilation equipment, the cooling plant, and the on-board electrical system. All these systems are modelled with regard to their power demand (either average or dependent on the diesel engine power output) and their mean relative switch-on times.

The algorithms controlling the train run are laid out to achieve the minimum traveling time. The choice of the notch is controlled by a complex submodel that is based on an
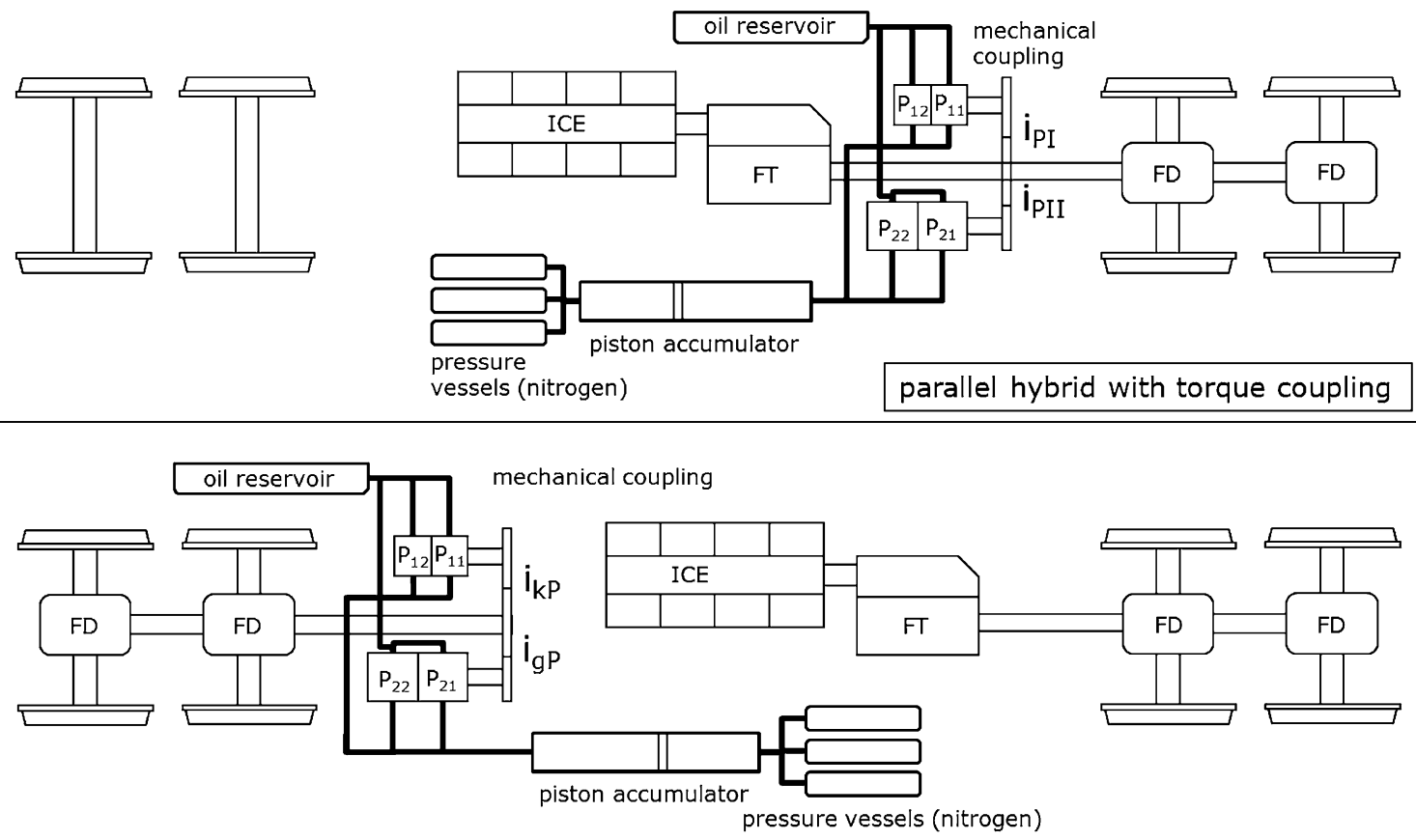

parallel hybrid with separated axles

\footnotetext{
$\mathbf{P}_{\mathbf{1 1}} / \mathbf{P}_{\mathbf{1 2}}$ - hydraulic pump, type I

$\mathbf{P}_{21} / \mathbf{P}_{22}$ - hydraulic pump, type II

ICE - internal combustion engine

FD - final drive

$\mathbf{i}_{\mathbf{P I}}$ - gear ratio of mechanical coupling for hydraulic pump(s) type I

$\mathrm{i}_{\text {PII }}$ - gear ratio of mechanical coupling for hydraulic pump(s) type II
}

Fig. 2 Possible parallel-hybrid layouts 
Fig. 3 Structure of the simulation model

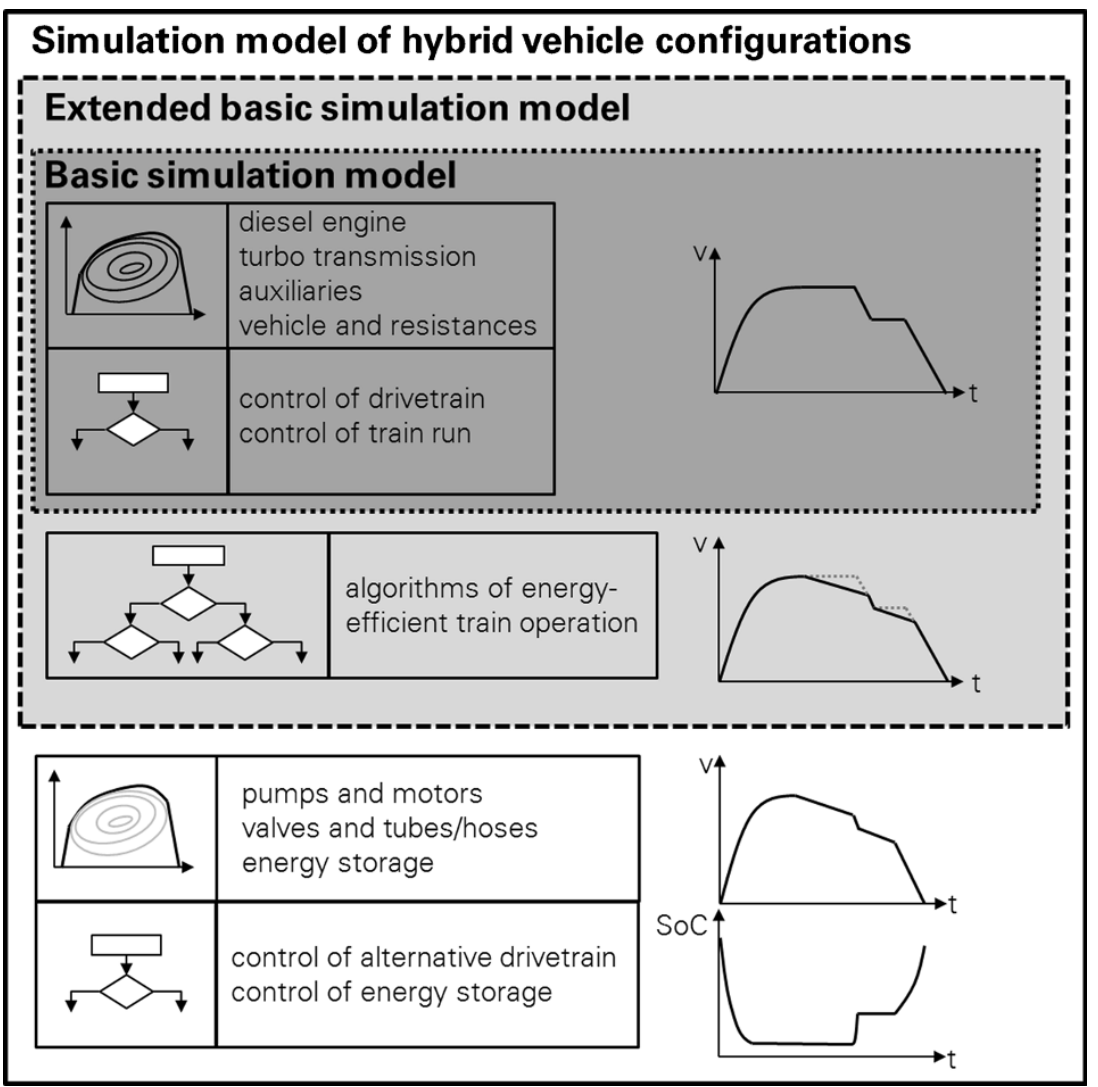

integral element and was inspired by the observations made on vehicles in real operation. The pattern of shifting the notches can be influenced by adjusting time constants and setting the limit for the maximum notch. The algorithm generates a saw tooth-like speed profile which is very common for these kinds of vehicles as there is generally no device installed to keep the vehicle speed constant after acceleration.

\subsection{Extended basic simulation model}

Applying driving strategies that are suitable to reduce fuel consumption has become common practice as drivers are specially trained to use the buffer times included in the schedules for coasting before starting to brake. To incorporate this practice into the simulation, the basic model was extended by a number of algorithms that automatically insert phases of coasting. The decision whether to switch off traction power or not is based on a rough prediction of the remaining travelling time that is compared to the scheduled time. The prediction is based on a linearised kinematic approach to calculate the times needed for coasting and braking. A reliable estimation of the average coasting acceleration along the relevant sections of the forthcoming route is therefore required. This acceleration is in turn dependent on the speed level (deceleration component due to vehicle resistance forces) and the gradients of the route (acceleration component due to track related resistance forces). The length of the route sections taken into account for estimating the coasting acceleration is altered in three preset steps (basic, prolonged, or shortened distance). The relevant distance is than automatically adjusted during simulation within the given limits depending on whether the route section ahead is predominantly ascending (short coasting distances), descending (long coasting distances), or flat.

The slope of the track and the line speed profile are also taken into consideration when fuel-efficient driving techniques are adopted so that steep downward slopes can be utilised to accelerate the vehicle. Moreover, short sections with increased allowable speed may be ignored as long as there is sufficient buffer time left.

The extended basic model allows simulating a driving style close to that of human drivers without using a computer-based assistance system. Thus the fuel consumption can be reduced but not optimised as this would require the program to do several iterations which prolong simulation time to an undesired extent.

The functioning of the submodels simulating an energyefficient driving style has been tested by comparing the simulated speed profiles and the calculated travelling times to those obtained through the software DYNAMIS [15]. This program is commercially used by train operating companies (e.g. the Federal Railway of Austria (OeBB)) and comprises a tool for the implementation of energy-efficient driving 
techniques. It has emerged that the submodel developed by the author was able to produce comparable results for a number of different operational cycles.

\subsection{Simulation model of the hybrid powertrain}

The basic model described above was further extended by the elements of the alternative drive train. These comprise the hydraulic machines, the valves, hoses and tubes, as well as the piston accumulator and the pressure vessels. As the standard models of hydraulic machines within the hydraulic libraries of the simulation environment did not meet the requirements, the author developed his own components using standard elements and efficiency maps to model the behaviour of the hydraulic pumps/motors. The modelling of these components was based on the assumption that axial piston pumps are employed. The volumetric and the mechanical efficiency are separately determined subject to rotating speed and pressure level. They are included within the pump/motor component via a leakage flow and a pressure drop, respectively. The characteristic decline of efficiency when small swash plate angles are set is also included within the efficiency maps.

The model of the energy storage is also based on standard elements and uses the real gas equation by Redlich-KwongSoave. The pressure drop due to the friction of the piston is calculated separately. All thermal exchanges with the environment are taken into account using a time constant. The calculated temperature of the gas is therefore of mere theoretical nature and does not reflect the complex temperature profile within real pressure vessels.

The model of the piston accumulator was successfully verified by comparing the simulation results to measured data obtained from Voith Turbo [16].

The inevitable system of valves, hoses, and tubes was originally modelled in detail but it turned out that the simulation process was slowed down significantly by this solution. Another approach was thus taken by isolating this part of the model and using it to investigate the transmission behaviour. The input pressure and the flow rate were systematically varied within the expected range and compared to the output values. This enabled the author to create a performance map for a simplified model replacing the original one. This measure was critical in keeping simulation times within a range of several minutes rather than hours.

The general technical specifications and the additional masses of the proposed hydrostatic hybrid system can be found in Table 1 and Fig. 4, respectively. This configuration represents a solution that aims at minimizing the additional mass of the alternative drive train. The required extra mass is 2,400 kg, i.e. $300 \mathrm{~kg}$ per wheelset considering a twin-section diesel railcar with an axle arrangement of 2'B'+B'2'. The energy content of the storage is relatively low $(0.9 \mathrm{kWh}$ in
Table 1 General specifications of the proposed hydrostatic hybrid equipment for a twin-section railcar (values apply for the entire vehicle)

\begin{tabular}{ll}
\hline Total mass of hybrid equipment & $2400 \mathrm{~kg}$ \\
Number of piston accumulators & 2 \\
Number of pressure vessels & 2 \\
Volume per piston accumulator & 1201 \\
Volume per pressure vessel & 751 \\
Number of motors/pumps, type I & 2 \\
Number of motors/pumps, type II & 2 \\
Maximum nominal power of pumps/motors & $1162 \mathrm{~kW}$ \\
Minimum operational system pressure & $90 \mathrm{bar}$ \\
Maximum operational system pressure & $320 \mathrm{bar}$ \\
Nominal energy content of pressure storages & $0,9 \mathrm{kWh}$ \\
\hline
\end{tabular}

total), yet sufficient to make a difference as the simulation results presented at the end of this paper show.

Hydraulic pumps/motors with two different dimensions are suggested to be installed simultaneously. The reason behind this approach is the limited rotational speed of pumps with a high displacement volume. The chosen pumps of type I and II cover different rotational speed ranges and feature different displacements so that the smaller pumps (type I) can provide power up to the maximum vehicle speed, whereas the bigger ones (type II) are only activated at lower speeds. This concept enables the system to recuperate energy while braking in steep gradients as well as while braking to comply with changes in line speed within the upper speed range. The control

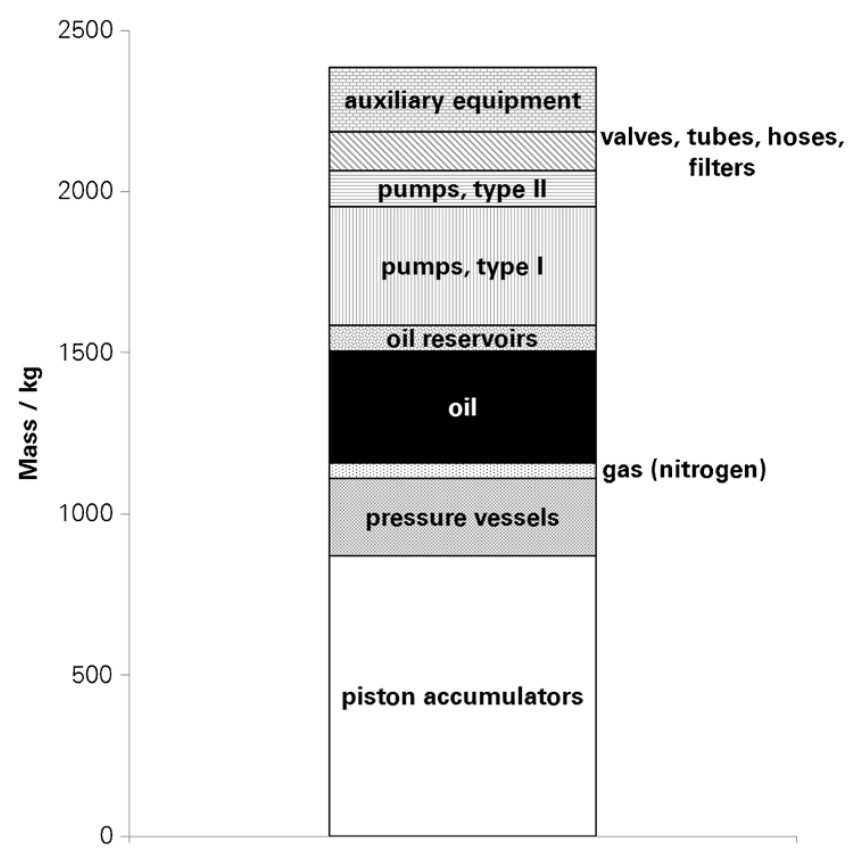

Fig. 4 Mass balance of the hydrostatic hybrid system 
algorithms of the hybrid drive train ensure that the pumps are switched off when their rotational speed-limit is exceeded.

\section{Simulation process}

The choice of suitable railway lines and the acquisition of route data are important issues when realistic results are to be obtained by simulation. The author has received data for eight different German railway lines that feature the operation of regional diesel railcars. The chosen branch lines comprise routes from all over Germany and reflect their different characteristics depending on the topographic circumstances. The data was provided by Deutsche Bahn AG and can be regarded as accurate and reliable. Table 2 contains the principal characteristics of the chosen lines with regard to their speed profile, gradients, and the distribution of stops or stations.

The simulation process is bound to a certain procedure in order to generate valid and realistic results (see Fig. 5). The first stage comprises the determination of the target travelling times. These are the result of two consecutive simulation runs using the (extended) basic simulation model which represents the conventional railcar. The first run aims at establishing the minimum travelling time between two stops. This time is consequently prolonged by $3 \%$ and serves as the target time henceforward. The overall travelling time is made up of the sum of the prolonged travelling times between the stops and the dwell times. The latter depend on the importance of a station and the number of passengers getting on or off the train. Based on observations on branch lines in Eastern Saxony, a period of 50 and $70 \mathrm{~s}$ was defined for stopping at minor or major stations, respectively. Having established the target travelling time, another simulation run is carried out (stage 2) with the algorithms for energy-efficient driving being activated. If the target time for the entire train journey is met within a range of $15 \mathrm{~s}$, the simulated values for travelling time and fuel consumption will be accepted as a reference for the hybrid model. Otherwise some of the parameters have to be adjusted and the simulation run needs to be repeated.

The third stage of the simulation involves the simulation model of the hybrid vehicle. At the beginning, the state variables of the energy storage (pressure, volume, and temperature) have to be determined by a reasonable estimation. A first simulation is then carried out for one direction of travel. At the end of the simulation a certain amount of time is reserved to represent the reversing time of the train. During this time, thermal exchange between energy storage and the environment causes both pressure and temperature drops of the storage gas until equilibrium is reached. A period of $10 \mathrm{~min}$ has been found to be sufficient to account for these processes. The values at the end of the simulated run for one direction are then used as initial values for the simulation of the train run in the opposite direction. Simulations for both directions have to be repeated until there are no longer any discontinuities within the time-dependent progress of the storage gas state variables. This way it is ensured that the energy balance of the storage is equalised and all thermodynamic cycles are closed.

The second parameter that has to be checked along with the state variables of the storage gas is travelling time. In order to properly judge the benefits of using an alternative drivetrain, it is essential not to mix the effects of energy-efficient driving and hybridisation. Therefore the simulated travelling times of the hybrid vehicle must not differ from the reference times by more than half a per cent. This implies that the buffer time (3\% of the shortest travelling time) is used by the conventional and the hybrid vehicle to the same extent. It is only then that two train runs are comparable. In case the simulated times differ significantly, the simulation has to be rerun with adjusted parameters of the submodels containing the algorithms for applying an energy-efficient driving style.

The simulation model of the hybrid vehicle is adjusted to different routes by changing the gear ratio of the mechanical couplings (see Fig. 2). The choice of suitable values mainly

Table 2 Statistic analysis of route data used for the simulation of hybrid railcar performance

\begin{tabular}{|c|c|c|c|c|c|c|c|c|c|}
\hline Line Number & & 1 & 2 & 3 & 4 & 5 & 6 & 7 & 8 \\
\hline Route length & $\mathrm{km}$ & 52,4 & 61,9 & 54,5 & 47,6 & 20,7 & 68,0 & 90,4 & 81,4 \\
\hline Average distance between two stops & $\mathrm{km}$ & 8,7 & 6,9 & 10,7 & 9,5 & 3,5 & 6,2 & 5,6 & 4,0 \\
\hline Standard deviation of distances between two stops & $\mathrm{km}$ & 3,6 & 3,0 & 4,8 & 2,9 & 0,6 & 2,1 & 2,2 & 2,0 \\
\hline Average longitudinal gradient & $\%$ & 0,1 & 0,0 & 3,1 & 3,2 & 3,6 & 0,7 & 0,2 & 2,4 \\
\hline Share of downhill line sections (with respect to route length) & $\%$ & 23,7 & 18,4 & 10,7 & 10,0 & 16,0 & 47,5 & 37,6 & 21,4 \\
\hline Share of flat line sections (with respect to route length) & $\%$ & 51,6 & 66,9 & 27,3 & 34,0 & 17,9 & 13,4 & 22,7 & 31,4 \\
\hline Share of uphill line sections (with respect to route length) & $\%$ & 24,7 & 14,7 & 62,0 & 56,0 & 66,1 & 39,1 & 39,7 & 47,2 \\
\hline Average allowable line speed & $\mathrm{km} / \mathrm{h}$ & 98 & 114 & 112 & 95 & 69 & 97 & 118 & 118 \\
\hline
\end{tabular}




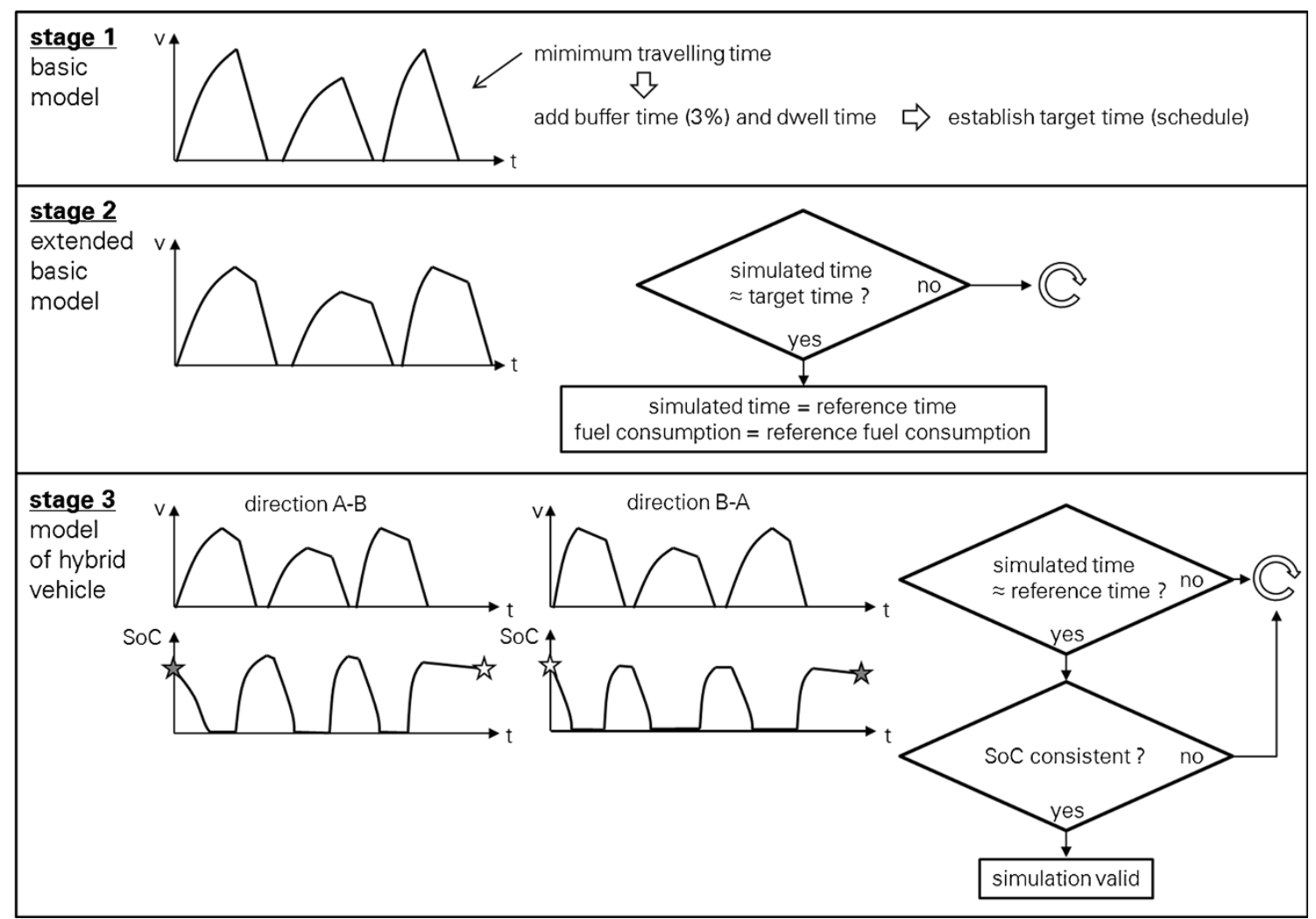

Fig. 5 Simulation process

depends on the speed profile of the line that determines the general speed level at which braking starts. The pumps must be able to transfer the entire oil volume from the reservoir to the accumulator during the braking process. Clearly, lines with very complex speed profiles or a generally high line speed level may require different gear ratios than those with low or very simple speed profiles.

\section{Simulation results}

The most important result of the simulation process is a prediction as to how much fuel is likely to be saved when the proposed hybrid system is installed on a given vehicle. Figure 6 shows the potential fuel savings for the railway lines characterised in Table 2 with respect to the fuel consumption of a conventional vehicle. Both the effect of applying an energy efficient operational strategy and the benefits of using the hybrid drive train are shown. The relation between the effects of both measures differs according to line profile and the direction of travel. The full potential shown in Fig. 6 can only be exploited in case there is no deviation from schedule as delays require train drivers to abandon energy-efficient operation and resort to a driving technique that minimises travelling time. As far as delayed journeys are concerned, a positive effect of hybridisation can be maintained even though coasting is reduced to a minimum. Simulations run by the author indicate that the fuel savings related to the use of the hybrid drive train are reduced by one to two thirds of the values calculated for train journeys without delays. The actual magnitude of the reduction depends on the kind and cause of the delay.

Despite the fact that the energy storage of the simulated hybrid configuration is comparatively small, reductions in fuel consumption by hybridisation can be expected to reach up to $16 \%$ (see Fig. 6). For the majority of lines, however, the hybrid-related reductions range between $5 \%$ and $10 \%$, whereas fuel saving driving techniques make up between $10 \%$ and $25 \%$. Most of the routes chosen for simulation do not show a flat or symmetric elevation profile, meaning the direction of travel influences fuel consumption. As coasting can be applied more extensively on line sections with descending slopes, the effect of hybridisation in these cases is generally stronger than on line sections with ascending slopes. Comparing lines 1 and 2 (with flat elevation profiles) to lines 3,4 , and 5 (all ascending in direction $\mathrm{A}$ and descending in direction $\mathrm{B}$, respectively) confirms this conclusion.

The most important factor having an impact on the hybrid-related fuel saving potential is the average distance between stops, as Fig. 7 underlines, where the data concerning this matter shown in Fig. 6 is rearranged. 


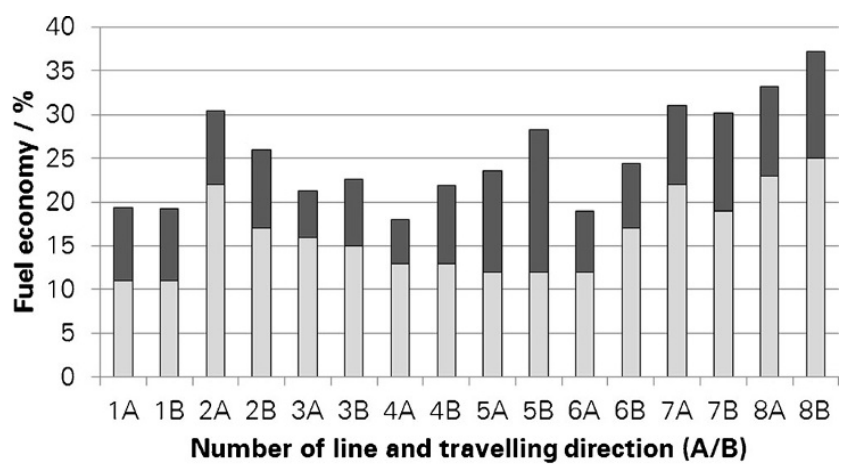

$\square$ Energy efficient train operation $\quad$ Hybridisation

Fig. 6 Simulation results concerning the predicted reduction of fuel consumption on different railway lines

The core message can be summed up by stating that recuperating energy is most beneficial when the average distance between two stops is relatively short. According to Fig. 6, line number 5 offers the highest potential for fuel savings, no matter which direction of travel is considered. This can be explained by taking into account that this line features an average station spacing of only $3,500 \mathrm{~m}$ in combination with a comparatively even distribution of stops along the route (see Table 2). In contrast, line number 3 exhibits the largest average station spacing in connection with the highest standard deviation of the distances between stops. This results in a significant reduction of possible fuel savings by hybridisation.

The hydrostatic system alone is able to accelerate the vehicle to a speed of about $18 \mathrm{~km} / \mathrm{h}$ on level track provided that the hydraulic accumulator has been sufficiently charged before. Covering distances between 100 and $500 \mathrm{~m}$ in zeroemission mode at the indicated speed level would require 30 to $130 \mathrm{~s}$. The outcome of this is a prolongation of the travelling time that might be unacceptable. It is therefore obvious that the energy content of the storage system presented is not sufficient for this kind of operational strategy.

Simulations showed further that the recuperated energy is utilised best in supporting the conventional drive train rather than substituting it. All the results shown in Figs. 6 and 7 were obtained for operation in boost mode using adhesion factors between wheels and rail of not more than 0.25. Simulation runs with reduced conventional power output during the operation of the alternative drivetrain resulted in fuel efficiencies inferior to those presented in Figs. 6 and 7.

In addition to the calculation of fuel consumption and journey time, simulations showed that the overall system efficiency of the considered hybrid drive train ranges between $45 \%$ and $60 \%$ depending upon the actual operational cycle. It can be raised above $60 \%$ by thermally isolating the gas of the energy storage, thus shifting the storage efficiency from around 85 to over $90 \%$.

\section{Conclusions}

A configuration for hybrid diesel railcars based on a comparatively small hydraulic system (total mass: 2, $400 \mathrm{~kg}$ ) is presented that could be applicable for existing vehicles as well as newly developed ones. Although the system described is inferior to electric systems regarding the amount of storable energy and overall system efficiency (cf. $[5,7]$ ), reductions in fuel consumption ranging between 5 and $16 \%$ can be expected. The simulation process described in this paper takes into account the effects of both fuel efficient driving techniques and hybridisation. The comparability and validity of simulation runs is ensured by strictly monitoring the simulated travelling times. In doing so, the particular effects of different measures on fuel consumption can be clearly distinguished. The characteristics of the railway line (station spacing, speed profile, gradients, and curves) are a major influence on the achievable fuel economy as the comparison of simulation results for eight different routes shows.

The average distance between two stops is the most important factor affecting the fuel saving potential of a hybrid vehicle followed by the gradients along the route. The overall efficiency of the hybrid drive train ranges between 45 and $60 \%$, but simulations showed that it can be enhanced above $60 \%$ by taking measures to thermally isolate the gas of the energy storage.

The simulations indicate that adding the power of the hybrid drive train to that of the conventional one (i.e. operation in boost mode) leads to the highest amount of fuel savings.

The results obtained for the considered system are encouraging, and therefore, further research on different hydrostatic hybrid configurations with higher energy content and power output of the alternative drive train will be conducted. Looking at twin-section vehicles with two diesel engines facilitates some interesting options, including the replacement of one engine by hybrid equipment.

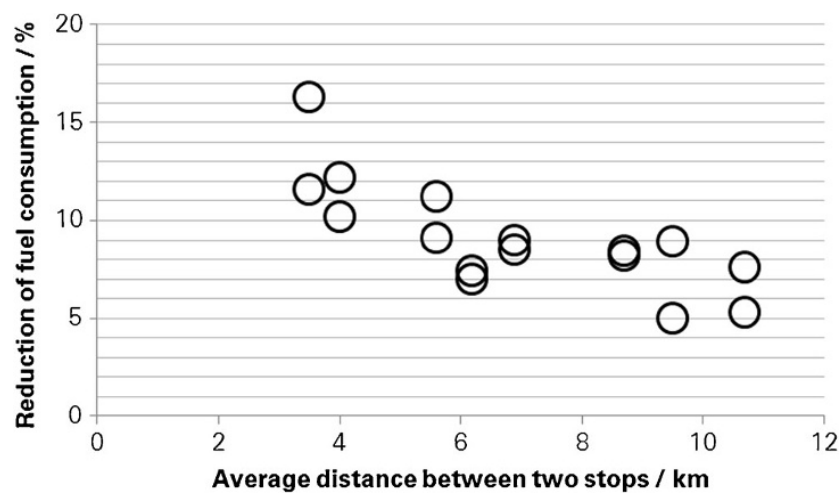

Fig. 7 Dependency of the fuel economy due to hybridisation upon the average station spacing 
The conclusion of this research project will include the modelling of parallel hybrid configurations using electric motors and electric double layer capacitors or batteries as energy storages.

Based on the findings of the simulations, an economic evaluation of the different hybrid configurations can be conducted to obtain a comprehensive idea of the options that occur when the hybridisation of diesel hydraulic railcars is considered.

Acknowledgments The author wishes to thank Mr. Uwe Steglich who contributed significantly to the basic simulation model described in the text by developing the submodels of the diesel engine and the fluid transmission as well as the control algorithms connected to these elements.

Open Access This article is distributed under the terms of the Creative Commons Attribution License which permits any use, distribution, and reproduction in any medium, provided the original author(s) and the source are credited.

\section{References}

1. Hillmansen S, Robert C (2007) Energy storage devices in hybrid railway vehicles: a kinematic analysis. Proc IME F J Rail Rapid Transit 221:221

2. Nick M (2002) Hybridantrieb mit Nutzung der Bremsarbeit bei Dieseltriebwagen. EI - Der Eisenbahningenieur 53(9):76

3. Lu S, Meegahawatte DH, Guo S, Hillmansen S, Roberts C, Goodman CJ (2008) Analysis of energy storage devices in hybrid railway vehicles. International Conference on Railway Engineering - Challenges for Railway Transportation in Information Age, 2008. ICRE 2008
4. Wen Q, Kingsley S, Smith RA (2007) Energy simulation of hybrid inter-city trains. Proceedings of the Institution of Civil Engineers, Vol. 160, Series: Energy, No. 3, pp 123

5. Neubauer M, Neudorfer H (2009) Simulation des Einsatzes neuer Technologien im Antriebsstrang eines dieselelektrischen Triebfahrzeuges. Elektrotechnik und Informationstechnik 126(4):19

6. Read MG, Griffiths C, Smith RA (2010) The effect of driving strategy on hybrid regional diesel trains. Proc IME F J Rail Rapid Transit 225:236

7. Dittus H, Hülsebusch D, Ungethüm J (2011) Reducing DMU fuel consumption by means of hybrid energy storage. Eur Transp Res Rev 3:149

8. Ihara $\mathrm{H}$ et al (2008) Development of motor-assisted hybrid traction system. Proceedings of the 8th World Congress on Railway Research, Seoul, 2008

9. Baseley S, Ehret C, Greif E, Kliffken M (2007) Hydraulic hybrid systems for commercial vehicles. SAE Technical Paper 2007-014150, doi:10.4271/2007-01-4150

10. Silvan E, Feyerabend L, Sachse C (2001) Abfallsammelfahrzeug x2eco mit hydraulischem Hybridantrieb, 3. Fachtagung Hybridantriebe für mobile Arbeitsmaschinen, Tagungsband 2001, Karlsruher Schriftenreihe Fahrzeugsystemtechnik, KIT Scientific Publishing

11. Thiebes $\mathrm{P}$ (2011) Hybridantriebe für mobile Arbeitsmaschinen, $\mathrm{PhD}$ Thesis, Karlsruher Institut für Technologie

12. Emadi A (2005) Handbook of automotive power electronics and motor drives. CRC/Taylor \& Francis, Boca Raton

13. Kache M, Steglich U (2011) Fahrdynamische Untersuchungen an dieselhydraulischen Triebwagen mittels 1D-Systemsimulation. ZEVrail 135(6-7):258

14. Kache M, Steglich U (2011) Betriebliche Aspekte bei der Simulation dieselhydraulischer Triebwagen. ETR - Eisenbahntechnische Rundschau 60(9):42

15. Radtke A, Monecke L, Müller L (1998) Dynamis: a model for the calculation of running times for and efficient time-table construction, Proceedings: Comprail 1998, Lisboa, Portugal

16. Discher A (2010) Prototyp zur hydrostatischen EnergieRekuperation bei Dieseltriebwagen, PhD-Thesis, TU Dresden 